\title{
3rd CMD General Conference
}

The first of the EPS General Condensed Matter Physics Conferences took place in 1980 in Antwerp, the second in 1982 in Manchester and the third from 28 to 30 March 1983 at the Ecole Polytechnique Fédérale de Lausanne. These Conferences are by now well established as indicated by the following numbers:

\begin{tabular}{lrrr} 
& $\begin{array}{c}1980 \\
\text { Antwerp }\end{array}$ & $\begin{array}{c}1982 \\
\text { Manchester }\end{array}$ & $\begin{array}{c}1983 \\
\text { Lausanne }\end{array}$ \\
\hline Participants & -600 & 581 & 623 \\
Inv. speakers & 63 & 59 & 66 \\
Plenary Session & 9 & 5 & 6 \\
Symposia + & & & \\
Gen. inter. & 11 & 12 & 15 \\
Sessions & 307 & 350 & 427 \\
Contrib. papers & & & \\
\hline
\end{tabular}

From the point of view of an organiser, there were no major problems with the Conference in Lausanne although the quality of projections in the large lecture hall "Salle Polyvalente" meant that some effort was required to follow the plenary sessions. Otherwise, the atmosphere was relaxed and it was easy to find people and also to find a quiet corner for discussions.

Two satellite meetings materialised. The one on "3rd Metallic Magnetism" is covered below, the second on "Impurity Induced Phase Transitions in $\mathrm{KTaO}_{3}$ " had been organised at the EPFL 25-26 March 1983. There have also been two official and several unofficial get-togethers during the Conference.

Students were treated as normal participants, but the Conference paid each a contribution of Sw.Fr. 300. - towards their living expenses; 44 made use of this provision. The Conference has also made its full contribution to the EPS Young Physicists Fund which now is voluntary.

Generally a conference cannot pay its participants. There are, however, always special cases and cases of particular hardship, and the organisers were very fortunate to have returned as a gift from a number of invited speakers from Switzerland, the living expenses paid to them by the Conference, with which they were able to help a number of foreign colleagues. The Organisers would like to express their profound appreciation to their Swiss colleagues.

A good habit which has been introduced is the organisation of an open discussion between the CMD Board and the CMD Members. The open meeting in Lausanne did not quite have the attendance it deserved, but it was quite informative nevertheless.

It is always difficult to judge with any certainty the success of a conference, but from the response received either orally or by mail afterwards, from colleagues numbering about $10 \%$ of the participation, it seems that at least two of the objectives of these General Conferences were attained, notably the creation of a European forum for discussions and the possibility of obtaining, with high efficiency, a representative overview of the field.

Abstracts of the papers have been published in the Europhysics Conference Abstracts series, Vol. 7B and in the following articles three topics have been picked out to give to the readers of Europhysics News a flavour of the rich field that is condensed matter physics today.
Last but not least the Organisers would like to thank the invited speakers and those who contributed to the running of the Conference and send all their best wishes to their colleagues organising the succeeding 4th General CMD Conference of the EPS, 19-23 March 1984 in Den Haag.

W. Czaja

\section{Localization and Interactions}

\author{
T.M. Rice, Zürich \\ (Theoretical Physics, ETH)
}

In the past few years there has been renewed impetus to consider the properties of electrons moving in a disordered medium. It is now over twenty years since Anderson showed in his classic work that a single electron becomes localized and will no longer diffuse if the random potential is sufficiently strong. However, reality is usually a finite density of electrons interacting with each other through the Coulomb interactions. This interaction cannot be treated as a weak perturbation on the single particle problem. For example, it is known that electrons in an ordered medium must have a discontinuous transition as a function of density due to the long range nature of the Coulomb interaction between them. Considerable progress had been made on the separate parts of the problem, but the key question of how the transition from the metallic phase to the localized insulating phases proceeds, remained without a definite answer either from theory or experiment.

The renewed interest and progress on this subject was sparked by a new approach to the problem of a single electron in a disordered medium by Anderson and his coworkers. In a paper in 1979 they (generally referred to by the whimsical title of "gang of four") introduced a singleparameter scaling theory for the problem. Their theory is expressed in terms of the scaling of the conductance of a finite sample with the length of the sample. The critical dimensionality is 2 . For dimensionality $\mathrm{d}$ $<2$, all states are localized, while for $d>2$ the states are extended at large values of the conductance but become localized with sufficiently strong disorder - a transition now called the Anderson transition. In two dimensions, $d=2$, there are logartithmic divergences even in the limit of large con- ductance which lead to the conclusion that there cannot be metallic conduction for $d$ $=2$ at $T=0$. This effect is generally referred to as weak localization. Such a conclusion was also obtained independently at that time by Wegner, who mapped the Anderson localization problem onto a field theory, whose scaling behavior was obtained by usual renormalization-group methods.

Shortly afterwards Altshuler, Aronov and Lee found that for $d=2$, there was a breakdown of the Landau Fermi liquid description of interacting electrons in the presence of the impurities. Previously it was generally believed that for $d=2$ and 3 the presence of impurities would not lead to any important modifications of the Landau Fermi liquid theory. However, Altshuler and co-workers showed that for $d=2$ there were corrections which diverged logarithmically and had coefficients of the same order as the divergent terms found by the "gang of four". Dr. Altshuler was invited to talk on this topic at the EPS conference. Regrettably he did not come - a fact which illustrates the difficulties that some of our colleagues continue to face despite the efforts made to normalize scientific relations.

At the EPS meeting a beautiful series of experimental results on two-dimensional films were presented by two speakers, G. Bergmann (Jülich) and $Y$. Bruynseraede (Leuven). In his talk, Bergmann presented studies on metallic films showing weak localization effects in the resistivity, and an appealing physical discussion of the single electron effects as an interference in the back scattering direction between conduction electrons which are split in pairs of waves. An external magnetic field introduces a magnetic phase shift of the 